\title{
Caching Routing Indices in Structured P2P Overlays *
}

\author{
Hailong Cai, Jun Wang \\ Department of Computer Science and Engineering \\ University of Nebraska, Lincoln \\ \{hcai,wang\}@cse.unl.edu
}

\begin{abstract}
Because of the omnipresence of node dynamic activities, large scale P2P systems built on structured overlays suffer high maintenance overhead and compromised routing performance. In this paper, we study the characteristics of $P 2 P$ node dynamic behaviors and present a novel routing indices caching scheme, called SORIC, which solves this problem by fully exploiting the round-trip pattern in node dynamic behaviors and heterogeneity among peers in the system. SORIC selectively caches routing indices of transient departed nodes in other relatively stable and capable nodes for two purposes. First, rejoin of the cached nodes is drastically simplified to $O(1)$ complexity, thus cutting off a large portion of system maintenance overhead. Second, caching routing indices of departed nodes minimizes the negative effects of node departures and rejoins, and thus enables the system to sustain an uninterruptedly high quality routing service.
\end{abstract}

\section{Introduction}

Compared with Gnutella-like unstructured P2P systems, structured overlays (or called DHTs) offer a logarithmically bounded lookup efficiency and good scalability, but may suffer high maintenance overhead and compromised routing performance, especially in a highly dynamic environment. Unfortunately, a significant feature of P2P systems is the omnipresence of node dynamic activities. As we will show by trace studies in Section 2, there are many instances of node departure and arrival back activities undergoing at a given time. While some peers (possibly, with a high-speed network connection like cable modem) stay in the system for a long time, many other peers leave and rejoin the system very often, especially when low bandwidth connections are used. This is reasonable because these nodes do not have enough bandwidth to stay online for a long time.

* This work is supported in part by NSF under grant \#CCF-0429995 and a University of Nebraska Lincoln Layman Fund.
However, current structured overlays do not take the round-trip (a round-trip activity is defined as a node's departure and its next arrival back) rhythm into consideration, though some new systems have been proposed to deal with node churn [6]. By studying current DHT systems, we notice that no matter what kind of routing geometry is formed, or routing algorithm adopted, all the query messages are forwarded by looking up some kind of routing indices that are initialized by an expensive node join procedure. Unfortunately, when a node departs or fails, its valuable routing information is also lost, and has to be setup from scratch when the node comes back to the system. While it is inevitable to run the join procedure for a brand new node, doing this for round-trip nodes that are regularly coming back incurs a lot of maintenance overhead $[5,7,10]$ that could be actually avoided. Furthermore, before the node departure/failure is detected and the recovery algorithm finishes, all the paths through this peer are invalid, thus compromising the routing quality and resulting in poor static resilience [3].

The above observations suggest that caching the routing indices of departed nodes could be helpful in both routing performance and system maintenance. A simple idea is to cache the routing indices in each node's local buffer when the node leaves the system. When the node comes back, the cached routing indices are restored from the local disk instantly rather than initialized by a lengthy node join process. Unfortunately, this scheme incurs two major problems. First, the cached routing indices are likely to be out-of-date when the node comes back, especially in a highly dynamic environment. The cost to repair these routing indices may offset or even outweigh the benefits obtained from the scheme. Second, the cached routing information of departed nodes is of no use to the system before the nodes come back. In short, this local caching scheme does not help much on system maintenance and routing performance. To develop an effective routing indices caching scheme, we have to 1) keep the cached routing indices upto-date; and 2) be able to use them to facilitate query routing during the node absent periods. 


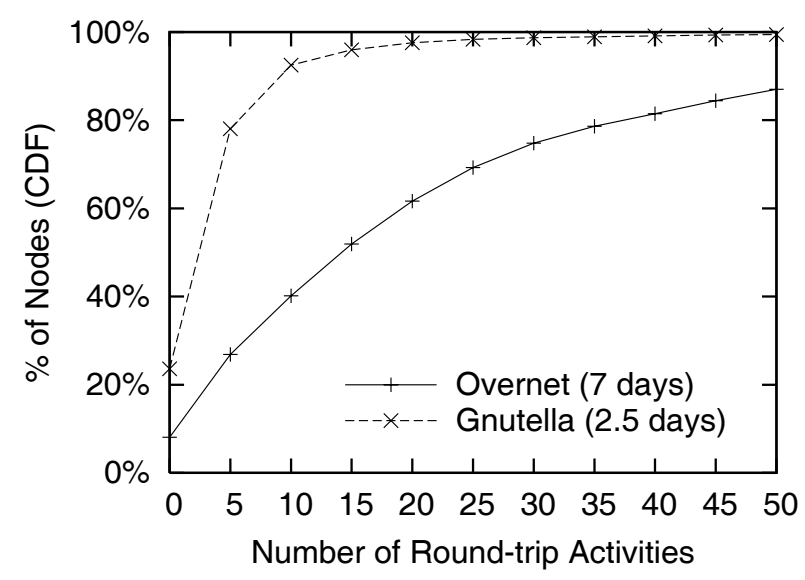

Figure 1. The CDF of the number of round-trip activities experienced by the nodes probed in the Overnet and Gnutella traces.

Another fact motivates us to develop a mature solution by taking advantage of node heterogeneity. The measurement study of Saroiu et al. [8] has shown that the node capacities may vary up to five orders of magnitude across the peers in the $\mathrm{P} 2 \mathrm{P}$ system. It is recommended to exploit the heterogeneity to improve system performance. Some researchers $[11,13]$ suggested using super-peers, which are supposed to form a secondary overlay, and forward/receive all the messages to/from the peers in each cluster. This approach is effective in reducing query hops, but complicates the design and implementation for the two-layer system architecture. Moreover, since super-peers have to forward much more messages than other nodes, they tend to become the network bottleneck or a point of failure for a whole group of peers.

By elegantly utilizing node dynamic activity patterns and node heterogeneities, we propose a lightweight routing indices caching scheme called SORIC (Structured Overlay with Routing Indices Caching) to address the abovementioned problems. SORIC selectively saves and maintains the routing indices of departed nodes on other live and relatively stable nodes during node departures, and the departed nodes may reclaim their cached routing indices upon arrival back. The cached routing indices can be used to uninterruptedly serve related queries on behalf of the departed nodes, thus sustaining a high quality routing service. In addition, when the departed nodes arrive back, they can quickly join the system by reclaiming their fresh routing indices. Notice that the selected caching host nodes do not have to be very powerful since they only perform additional indices caching and message forwarding for departed nodes. Our simulation results show that SORIC drastically reduces the maintenance overhead and boosts the routing performance compared to current structured P2P systems.

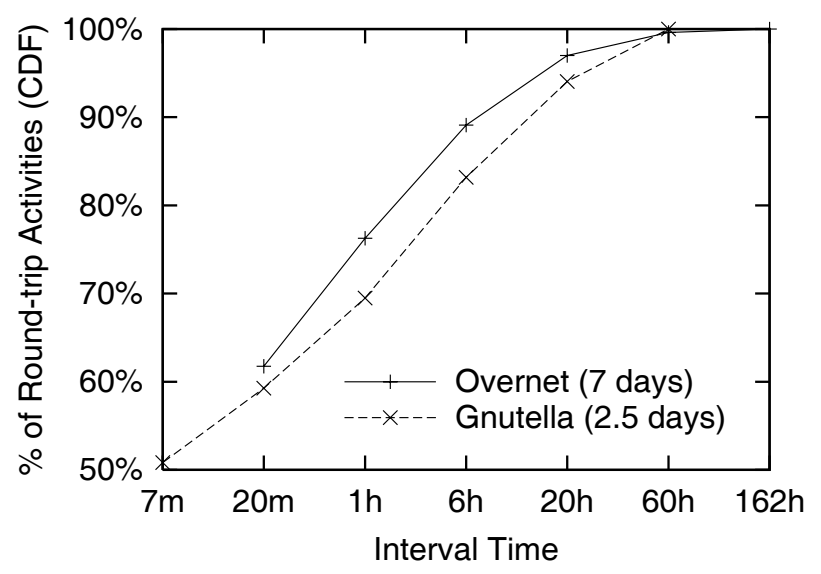

\section{Figure 2. The CDF of the interval times of the round-trip activities in the Overnet and Gnutella traces.}

\section{Node dynamic activities in DHTs}

A lot of measurement studies have shown the presence of many node dynamic activities in P2P systems $[1,8,9]$. We get the Overnet trace from University of California, San Diego [1] and the Gnutella trace from University of Washington [8] and study the characteristics of node behaviors in current $\mathrm{P} 2 \mathrm{P}$ systems.

The Overnet trace monitors 2,400 nodes in the network by probing them every 20 minutes and recording their arrival and departure events over a period of one week. This trace contains 27,151 node joins, among which 25,964, more than $95 \%$ are due to arrival back of nodes that have ever joined the system at least once. From the Gnutella trace collected by probing 17,125 peers every 7 minutes for a period of 60 hours, we derive 39,001 node arrivals, among which 31,399 , over $80 \%$ result from node rejoins. Due to limitation of the peer probing method, we are able to derive a node join event by its liveness and a node leave event by its unreachability, but can not tell a node leave is a graceful departure or unexpected node failure.

Figure 1 shows the cumulative distribution of the number of round-trip activities experienced by the nodes in both traces. It can be seen that over $92 \%$ Overnet nodes leave and rejoin the system at least once, and around $60 \%$ experience 10 or more round-trips within one week. In the Gnutella trace, while more than $77 \%$ peers have at least one rejoins, nearly $8 \%$ experience 10 or more round-trips within two and a half days. Both traces exhibit an obvious round-trip pattern among the participating nodes, while the Overnet trace sees more such activities than the Gnutella trace because of its smaller number of probed nodes and longer period of trace time. This also implies that in a typical P2P system, a majority of nodes will have more and more round-trip activ- 
ities over time. Additionally, we plot the cumulative distribution of interval times of round-trip activities in these two traces, as shown in Figure 2. The interval time is calculated as the time elapsed between a node departure and its next arrival back. From this figure we can see that more than $61 \%$ Overnet round-trips are done within 20 minutes, and nearly $76 \%$ in 1 hour. In contrast, over $50 \%$ Gnutella round-trips complete within 7 minutes, and around $70 \%$ within 1 hour.

The results indicate a large number of round-trip activities undergoing on many peers within a short time. This will result in extremely high system overhead because of the $O(\log N)$ complexity in DHT maintenance. Such workload may easily overwhelm those nodes with low bandwidth connections as well as apparent round-trip patterns. Furthermore, the routing performance may be seriously compromised if all of these node departures and rejoins are to be handled by conventional maintenance procedures used in current DHTs [3, 4].

\section{SORIC preliminaries}

Current DHTs discard the routing indices any time a node leaves the system, and have to run the lengthy node join process (including bootstrapping) when the same node arrives back. Instead of this, SORIC selectively caches the routing indices of departed nodes with frequent round-trip patterns in order to reduce the system maintenance overhead and improve the routing performance.

SORIC classifies participating node into three categories: RICH (routing indices caching host) nodes, RICC (routing indices caching client) nodes and open nodes. RICH nodes are peers ready to provide caching service to nodes in their service areas, while a RICC node refers to a client that may request the service from the RICH node to which it has registered. Those peers out of service areas of any RICHs are called open nodes. The service area of a RICH node is determined by service size and service radius. The service size defines the maximum number of RICC nodes a RICH can serve, and the service radius limits the maximum distance between a RICH node and its RICC nodes. Both parameters are used to avoid overloading RICH nodes and ensure good network proximity.

The three types of nodes have different characteristics as follows. In order to provide the caching service, $\mathrm{RICH}$ nodes have to be relatively stable and capable for this duty. It is expected that stable nodes in P2P systems tend to be relatively powerful, because otherwise they would not stay in the system for a long time concerning resource consumption. Although each RICC is serviced by a RICH node, it may be qualified to be a RICH candidate by itself or not. When the original RICH leaves or fails, a qualified candidate will take the responsibility and retain the service. The open nodes are not qualified to be RICH nodes because otherwise a qualified open node will work as a new RICH node itself. To determine the qualification of a node to be a RICH, we define a RICH candidacy factor for each node according to its availability and capacity. To be a RICH candidate, a node must have a RICH candidacy factor larger than a predefined threshold.

When departing the P2P system, a RICC node always sends its routing indices to its RICH node, asking for a caching service. If possible, the routing indices will be saved and maintained by the RICH node. When the same RICC node rejoins some time later, it may reclaim its fresh routing indices from the RICH and finish its join process in $O(1)$ time, rather than running an $O(\log N)$ join procedure as in most DHTs. During its absence, the RICH node maintains the cached routing indices and provides additional routing service to other nodes using the cached routing information, as if the departed RICC node is still alive. Furthermore, since the cached routing information is still available to be accessed, there is no need for other nodes to repair corresponding routing index entries that refer to this node. As a result, the negative affects of node round-trip activities are diminished by maintaining and utilizing the up-to-date routing indices of departed RICC nodes.

For the indices caching service, SORIC introduces several new data structures. Each RICH node maintains a routing indices cache, and a client list. The routing indices cache is the block of memory where routing index records of departed RICC nodes are cached. The client list contains some information for the live and cached RICC nodes in the RICH's service area, including the node status (live or cached), node RICH candidacy and estimated offline period (EOP) for those cached clients. To implement SORIC on top of structured overlays, for each routing table entry node $N$, we add the IP address of $N$ 's RICH node in addition to $N$ 's own IP address. A query message whose next hop is supposed to be node $N$ will be redirected to $N$ 's RICH when node $N$ is gone, as explained in later sections.

In addition to the above data structures, we define some new types of messages here. We explain more details in later related sections.

RICH Contact When departing and rejoining the system, a client node will contact the RICH by sending a "RICH Contact" message to get its routing indices cached and reloaded respectively.

Live Refresh These messages are sent from RICC nodes to their RICHs periodically, to keep the client lists on the RICHs up to date. A timer is attached to each item in a client list. Initially the timer is set to a slightly larger value than the "Live Refresh" cycle time. To keep the list up to date, each live RICC node sends "Live Refresh" messages to its RICH periodically. When receiving a refresh message, RICH node updates the corresponding item in the list and resets the timer. At the same time, the RICH node 
replies the refresh message instantly, so that a RICH failure can be quickly detected by its client nodes through an acknowledgement timeout.

RICH Change When a departed RICH node is replaced, the new RICH node that inherits the client list will send a "RICH Change" message to the clients.

RICH Ready When a RICH is replaced, the new RICH may broadcast a "RICH Ready" message within its service radius to cover open nodes until there is no reachable open nodes or the service size is reached.

Reverse Update The RICH information integrated into the routing entries may be stale if the RICH of that node is changed. We employ a lazy reverse update mechanism to solve the problem during normal query routings.

\section{SORIC algorithms}

In this section, we first present the self-organization algorithms in SORIC, and then propose an enhanced routing procedure that exploits the cached routing indices to sustain a high quality routing service.

\subsection{RICH selection}

When a RICH candidate enters the system, it becomes a new RICH if it can not find an existing RICH node nearby that can provide caching service for it. After becoming a new RICH node, it broadcasts a "RICH Ready" message within service radius. All open nodes that receive this message will reply with positive ACKs. Based on the replies, the RICH accepts RICC nodes with a first-come-first-serve policy until the service size is reached.

When the RICH is on a planned leave, it will try to pick up the next most qualified node among its live RICC nodes to become the new RICH node. If no RICH candidate is found in the list, all the live RICC nodes will become open nodes consequently. If such a candidate is found successfully, this node becomes the new RICH and inherits the client list. However, the cached routing index records and corresponding items in the client list are both discarded since these nodes can not find the new RICH node when they come back. After that, the new RICH sends a "RICH Change" message to the live RICC nodes, which will change their RICH addresses accordingly. If the service size is not reached, the new RICH will then broadcast a "RICH Ready" message to cover open nodes nearby.

In case of a RICH failure, as indicated by an acknowledgement timeout of a "Live Refresh" message from a RICC node, there is no chance for the failed RICH to pick a new one. If the client that detects the failure is not a RICH candidate, it simply becomes an open node. If it is a candidate, it will become a new RICH and broadcast a "RICH
Change" message along with the old RICH IP within its service radius. Upon receiving this message, each live RICC node compares its RICH IP with the one in the message, and if they are the same, switches to the new RICH node after being acknowledged by the new RICH. In addition, the open nodes in this service area will also be covered by this RICH node similarly.

However, this strategy may lead to multiple RICH nodes rising up after a RICH failure. Suppose $R_{1}$ is the first RICH candidate to detect the RICH failure event, if another RICH candidate also detects the RICH failure by a refresh acknowledgement timeout before it receives the "RICH Change" message from $R_{1}$, it may become a new RICH node unnecessarily. Fortunately, the probability of this case is very low. Let $T$ be the cycle time of "Live Refresh" messages. Assume a RICH serving a number of RICC nodes, $X$ of which are qualified to be RICH, and at time $t$ RICH candidate $R_{1}$ firstly detects the RICH failure event. To claim a new RICH, $R_{1}$ sends out the "RICH Change" messages within the service radius $r$. These messages will reach all potential RICC nodes of this new RICH node within time $r$. Suppose the start time for each RICC node to send next refresh message is randomly distributed, the probability for $i$ nodes to detect the RICH failure simultaneously (i.e. from time $t$ to time $t+r$ ) is

$$
p_{i}=\left(\begin{array}{c}
X-1 \\
i-1
\end{array}\right)\left(\frac{r}{T}\right)^{i-1}\left(1-\frac{r}{T}\right)^{X-i} .
$$

Suppose the service size is 40 , service radius is $r=30 \mathrm{~ms}$, and the cycle time for refresh messages is $T=10$ minutes. Even if all live clients are RICH candidates, i.e., $X=40$, the maximum probability of initializing two RICHs $(i=2)$ is calculated as $p_{2}=\left(\begin{array}{c}40-1 \\ 2-1\end{array}\right)\left(\frac{30 m s}{10 m i n}\right)^{2-1}\left(1-\frac{30 m s}{10 m i n}\right)^{40-2}=$ $0.1946 \%$. Considering all possible scenarios, the total probability for two or more nodes to become RICH nodes is

$$
\begin{aligned}
p_{\text {all }} & =\sum_{i=2}^{X} p_{i}=1-p_{1}=1-\left(1-\frac{r}{T}\right)^{X-1} \\
& =1-\left(1-\frac{30 m s}{10 m i n}\right)^{40-1}=0.1948 \% .
\end{aligned}
$$

Since the propagated latency within a service radius is much shorter than a refresh cycle, the live RICC nodes are very likely to see the "RICH Change" message from $R_{1}$ before they find the RICH failure. In most cases, most live RICC nodes in the old service area will successfully migrate to the service ares of new RICH $R_{1}$ that is the first RICH candidate to detect the RICH failure.

\subsection{Node join, departure and failure}

When joining the system, a new node $\mathrm{P}$ that doesn't have a RICH IP address in its local buffer still has to run the DHT 
bootstrapping and join procedure to collect its routing information. After this, node $\mathrm{P}$ contacts its neighbors to see if any RICH node is available. These neighbors will reply with their RICH IP addresses if they have. Then node P sends contact messages to these RICHs requesting for service coverage. On receiving a positive reply from a RICH node, node $\mathrm{P}$ successfully becomes a client of that RICH node. Following replies are simply ignored. However, in case of no RICH to contact or positive replies, node P may become a new RICH node if it is qualified, or otherwise stay as an open node.

Having RICH IP address in local buffer, a rejoining node firstly tries to contact the RICH node and reclaim its routing indices. If the node successfully gets back its routing indices from the RICH node, this process is called a fast rejoin done instantly. At the same time, its node status, EOP value and RICH candidacy are updated on the RICH node accordingly. However, if the reclaim fails for some reasons (the RICH has changed, or the cached routing index has been replaced), this node has to run a slow rejoin process, which is the same as a new node join.

In the simplest case, open nodes depart the system autonomously. For a live RICC node to depart or fail, we have the following three cases.

First, a live client departs the system intentionally, and its routing indices are not cached because the cache is full and no victim entry is found. This implies that the departing node is not likely to arrive back in near future, so the RICH node simply removes it from the client list.

Second, a live client departs the system intentionally, and its routing indices are successfully cached (its status is changed in the RICH's client list accordingly). Because a cached node can not send refresh messages, its timer is changed to a slightly larger value than its EOP. This is straightforward since the cached node is expected to rejoin the system within its EOP.

Third, a node fails silently. If the timer of a live client expires before RICH receives a refresh message from it, this node is assumed to have failed. In this case, SORIC simply removes the corresponding item from the client list. When coming back, this node will be regarded as a new node. With a more aggressive caching scheme, live RICC nodes can send routing indices to their RICH nodes periodically (e.g. along with the refresh messages), such that routing indices of these nodes may be successfully cached on their RICH nodes before failures.

\subsection{Routing indices cache replacement}

If the cache is full when a RICC node departs, then we have to decide whether to discard this node's routing information or free a space in the cache. An approximately LRU replacement algorithm is developed to manage the routing indices caches so that RICC nodes most likely to come back sooner will get cached. To do this, we design an Estimated Offline Period field (EOP for short) for each node to indicate its offline period before its coming back next time. Each node is assigned a default value of EOP when it joins in the system for the first time, and updated each time the node rejoins the system by the formula $E O P_{n e w}=E O P_{\text {old }} \times \alpha+O P \times \beta$, where $\alpha+\beta=1$, and OP is the offline period since the node's last departure. The values of $\alpha$ and $\beta$ indicate how much the node's latest round-trip activity affects its EOP. Typically we set $\alpha=0.2$, $\beta=0.8$, as suggested by our experiments, but they may be adjusted according to the amount of node round-trip activities observed.

When a RICH node receives a caching request from a departing RICC node, it checks if the cache is full or not. If it is full (if not, no replacement is needed), the RICH will examine the cache content seeking for a victim. If an "expired" record or an item of a node whose remaining offline time is larger than the departing node's EOP is found, this record becomes the victim for replacement. A node's remaining offline time is calculated by subtracting the elapsed offline time from its EOP value. The elapsed offline time can be obtained from the corresponding timer in the routing indices cache. If no such item is found, however, no replacement occurs as the departing node has the least probability to arrive back in the near future.

\subsection{SORIC routing}

When a peer leaves the system, SORIC tries to cache its routing indices. The cached information can still provide routing service on behalf of the departed nodes, making the offline node virtually online to the rest of the system. Unless the query is asking for an object stored on the departed node, the departure of a node whose routing indices are cached seems transparent to the query initiator and no routing indices repair is needed, i.e., its negative impact is minimized. Notice that the cached routing indices are continuously maintained by the RICH in a similar way to the query processes. A maintenance message may be directed to a RICH if the next hop node is cached in that RICH. The idea is to keep the routing information fresh until it is claimed by the rejoining node.

During the query lookup process, if the next hop node $\mathrm{N}$ is not alive, the previous node will set a "Cache Fetch" flag in the query message and send it to N's RICH node specified in this routing entry (if not null). Upon receiving this "Cache Fetch" tagged query message, the RICH node looks up in its cache for the corresponding nodeId and routing indices. If the information is found (cache hit), the query message simply bypasses the offline node and still goes through the optimal route. If the routing information is not found 


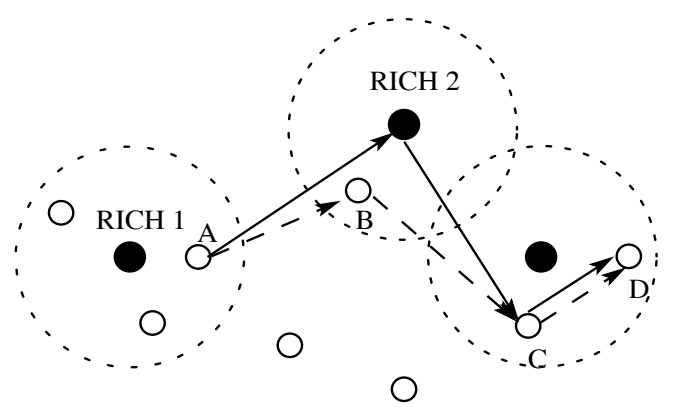

Figure 3. Routing of a query message from node $A$ to node $D$. Dashed lines show the route when node $B$ is online. Solid lines show the route when node $B$ is offline but its routing indices are cached in RICH 2.

in the cache (cache miss), this route fails and traces back to the last step. Our experiment results have shown a more than $90 \%$ cache hit rate when replaying both Overnet and Gnutella traces.

An example of the enhanced routing in SORIC is shown in Figure 3. Supposing node A issues a query with a key closest to node D's nodeId, and the optimal route is from A to $\mathrm{B}$ to $\mathrm{C}$ to $\mathrm{D}$. When node $\mathrm{B}$ leaves, its routing information is saved in the routing indices cache of RICH 2, assuming it is cached successfully. Now suppose the same query message is sent from node A again. According to the lookup protocol, the next hop is node $\mathrm{B}$, which is now offline. Instead of trying another sub-optimal route, the message is sent to RICH 2. By looking up in the cache, RICH 2 finds node $\mathrm{B}$ 's routing indices in the cache and routes the message to the next hop, node $\mathrm{C}$.

When a RICH node is replaced, the RICH address in some other nodes' routing entries may become stale. We apply a lazy reverse update mechanism to solve this problem in the process of query routings. When forwarding a lookup message to the next hop node $\mathrm{N}$, the previous node always piggybacks N's RICH information to the message. On receiving this message, node $\mathrm{N}$ checks whether the attached RICH information is fresh or not. If not, it sends a "Reverse Update" message to the previous node on the route, which will make necessary corrections accordingly. Taking the same example above, suppose that RICH 2 is replaced by another candidate RICH 3, but B's RICH address in node A's routing table still refers to RICH 2. This inconsistency is resolved by our reverse update: when forwarding a query to node B, node A appends B's currently known RICH address in the message. Upon reception, B discovers this inconsistency and sends a "Reverse Update" message back to node A, and A can update the routing table entry immediately. Experimental results show that this scheme only leads to minor overhead.
Table 1. Maintenance overhead of Pastry and SORIC when running the two traces.

\begin{tabular}{|c|c|c|c|c|}
\hline Trace & \multicolumn{2}{|c|}{$\mathrm{msg} /$ (node $\times$ minute) } & Overhead & $\begin{array}{c}\text { Rejoin } \\
\text { reduction }\end{array}$ \\
\cline { 2 - 3 } & Pastry & SORIC & rate \\
\hline Overnet & 1.1891 & 0.2133 & $82.06 \%$ & $85 \%$ \\
\hline Gnutella & 0.9008 & 0.3441 & $61.78 \%$ & $84 \%$ \\
\hline
\end{tabular}

\section{Evaluation}

We developed a SORIC system simulator based on a representative DHT, Pastry and its latest prototype, FreePastry 1.3 [2]. Then we conduct trace-driven simulations to evaluate its benefits by replaying two real-world P2P traces, Overnet and Gnutella as mentioned in Section 2.

We built the simulator over a large-scale, physical network topology using the GT-ITM [12] model with 100,000 physical machines arranged hierarchically, from which $\mathrm{P} 2 \mathrm{P}$ nodes were randomly selected as required by the Overnet and Gnutella trace. In all the experiments, we set the Pastry parameters as $b=4,|L|=16$ and $|M|=32$. The default values for SORIC system parameters are set as follows: the cache size is set to 20 , service size 40 , and service radius 30 $\mathrm{ms}$. The default EOP is set to 6 hours so that most roundtrip activities can benefit from our SORIC caching scheme. The prototype was written in Java.

\subsection{Maintenance overhead reduction}

In order to measure the overall maintenance efficiency of SORIC, we use two specific metrics on maintenance overhead hereafter: overhead reduction and rejoin hit rate. The overhead reduction is calculated as the percentage reduction in total maintenance cost. The rejoin hit rate is the percentage of fast rejoins among all the rejoin events, and tells how well the routing indices cache replacement algorithm works. In experiments, we replay the Overnet and Gnutella traces using Pastry and SORIC algorithms, and collect the total number of maintenance messages and the number of fast rejoins and slow rejoins. Then we calculate the average number of maintenance messages per node per minute, the overall maintenance overhead reduction and the rejoin hit rates, as shown in Table 1. It shows that SORIC drastically reduces the system maintenance overhead by nearly $82 \%$ for Overnet, and $62 \%$ for Gnutella. The rejoin hit rate is around $85 \%$ and $84 \%$ respectively. The results also indicate that more benefit can be achieved as in the Overnet trace where nodes involve more round-trip activities. The reason is that SORIC has more chances to cache routing indices for departed nodes with more round-trip activities.

To examine the workload on different type of nodes, we collect the detailed numbers of messages used to process 


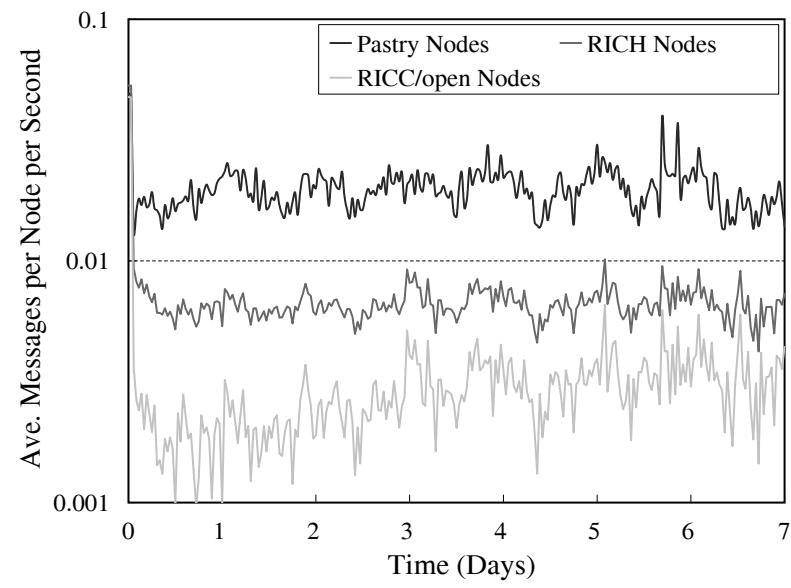

Figure 4. Maintenance overhead in average number of messages per node per second when replaying Overnet trace.

each node join and departure request in both traces, and plot the workload distribution among SORIC nodes compared to Pastry nodes over time, as shown in Figure 4 and Figure 5, respectively. The results demonstrate running SORIC on the Gnutella trace reduces the maintenance overhead for RICC and open nodes by a factor of 4 , while only increasing load on a small number of RICH nodes. This load increase is not significant with regarding to the bigger node capacity of RICH nodes over other nodes. For the Overnet trace, however, the workloads on both RICH and RICC/open nodes are significantly reduced, because there are much more beneficial round-trip activities involved in this trace so that maintenance overhead is substantially reduced on all participating nodes. This also implies that the more extensively peers conduct dynamic activities, the more maintenance overhead can be reduced by SORIC. This helps improve the system scalability in terms of the number of nodes leaving the network and joining back. Another easy observation is that the RICH nodes deal with more workload than RICC and open nodes. This skew of workload distribution approximately conforms to the heterogeneity among participating nodes, since the RICH nodes are supposed to be relatively stable and capable.

In the next experiment, we study the SORIC selforganization efficiency when some nodes fail unexpectedly rather than depart intentionally. Since failed nodes do not have a chance to be cached, the performance is expected to degrade, as is shown in Figure 6. Notice that when all node departures are changed to node failures, SORIC degrades to the baseline system and shows no improvement. In a system with failure rate less than $10 \%$, SORIC reduces the system maintenance overhead by more than $70 \%$ when running the Overnet trace and 53\% when running the Gnutella trace.

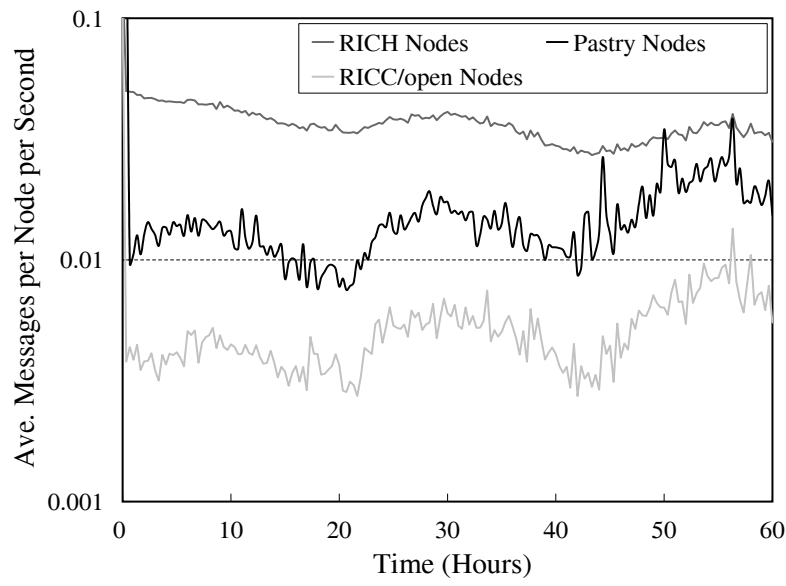

Figure 5. Maintenance overhead in average
number of messages per node per second
when replaying Gnutella trace.

\subsection{Routing performance improvements}

The routing performance is measured in terms of three metrics: propagated latency, logical hop and relative distance. We studied the routing performance of SORIC compared to Pastry by running lookup tests on several selected time instants during the trace-replay period. Specifically, we run 10,000 lookups for every 10,000 events in each trace consumed (i.e., replayed) by Pastry and SORIC. Then we collect the routing performance gains on propagated latency, routing hops and relative distance at each selected time instant, and calculate the averages for them.

As shown in Figure 7, SORIC offers substantial improvement on routing quality for both traces. This stems from the different approaches in Pastry and SORIC dealing with a query that passes an offline node on its optimal routing path. In Pastry as well as other DHTs, the query will be traced back to the last step. But in SORIC, the query will typically be forwarded to a RICH with "Cache Fetch" tag. If this request is satisfied (a routing cache hit), the query is further forwarded along the optimal route. Since a larger portion of nodes involve round-trip activities and have their routing indices cached with the Overnet trace, we can see more routing performance gains than Gnutella. Another metric to measure routing performance gains is the routing cache hit ratio, which tells how well the routing indices cached in RICH nodes answer the cache fetch requests for query messages. In the experiments, we obtain the number of routing cache hits and misses, and figure out a cache hit ratio of around $90 \%$ for both traces (not shown in the graphs for space limitation). This high hit ratio also implies a much improved fault resilience [3] due to more valid routing information maintained in the whole system. 


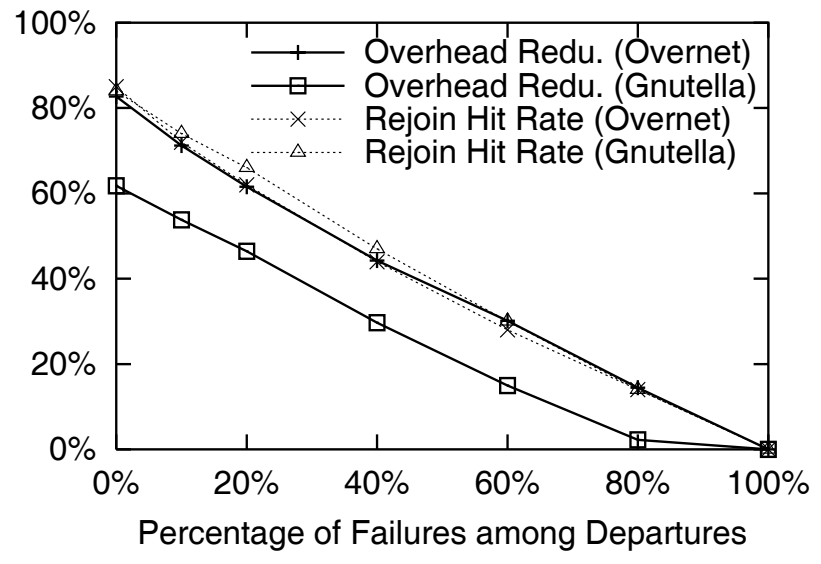

Figure 6. Maintenance overhead reduction and rejoin hit rate of SORIC with node failures.

\section{Conclusions and future work}

In this paper, we design and implement SORIC, a novel routing indices caching system that significantly reduces system maintenance overhead and improves routing performance for structured P2P systems in a dynamic environment. We advocate exploiting both node heterogeneity so as to assign different responsibilities to nodes with diverse capabilities, and the round-trip characteristics of dynamic activities in P2P systems by saving and maintaining routing indices for departed round-trip nodes. We find that SORIC drastically reduces the maintenance overhead by up to $82 \%$ while simultaneously boosting the routing quality when deployed on top of current structured P2P systems. In the future work, we will enhance our scheme in handling node failures, and implement it on top of other DHTs such as Chord and CAN and study its effectiveness and efficiency.

\section{References}

[1] R. Bhagwan, S. Savage, and G. Voelker. Understanding availability. In Proceedings of the 2nd International Workshop on Peer-to-Peer Systems (IPTPS), Berkeley, CA, Feb. 2003.

[2] FreePastry. http://freepastry.rice.edu.

[3] K. Gummadi, R. Gummadi, S. Gribble, S. Ratnasamy, S. Shenker, and I. Stoica. The impact of DHT routing geometry on resilience and proximity. In Proceedings of ACM SIGCOMM'03, pages 381-394, Karlsruhe, Germany, August 2003.

[4] D. Liben-Nowell, H. Balakrishnan, and D. Karger. Analysis of the evolution of peer-to-peer systems. In Proceedings of 21st ACM Symposium on Principles of Distributed Computing (PODC 2002), pages 233-242, Monterey, CA, USA, July 2002.

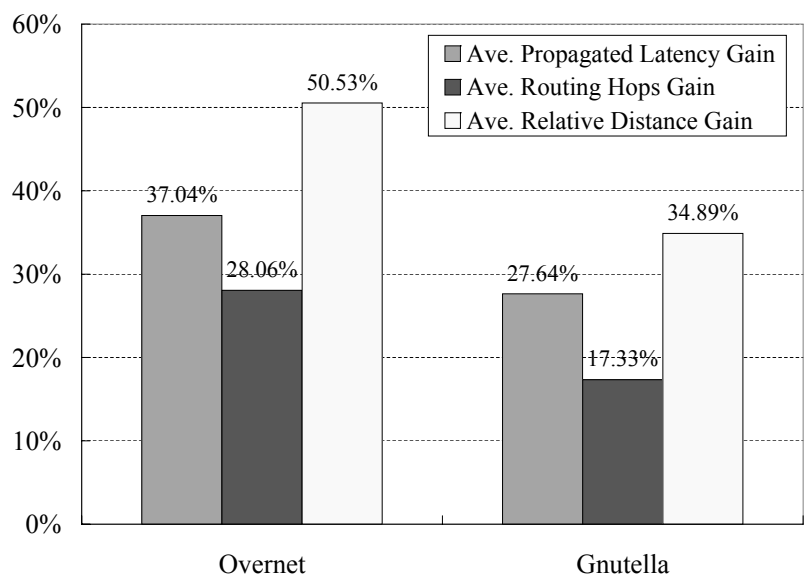

Figure 7. SORIC Routing performance gains compared with Pastry when running lookups.

[5] S. Ratnasamy, P. Francis, M. Handley, R. Karp, and S. Shenker. A scalable content-addressable network. In Proceedings of the ACM SIGCOMM 2001, pages 161-172, San Diego, CA, USA, August 2001.

[6] S. Rhea, D. Geels, T. Roscoe, and J. Kubiatowicz. Handling churn in a DHT. In Proceedings of the USENIX Annual Technical Conference, pages 127-140, Boston, MA, USA, June 2004.

[7] A. Rowstron and P. Druschel. Pastry: Scalable, decentralized object location, and routing for large-scale peer-to-peer systems. In Proceedings of the 18th IFIP/ACM International Conference on Distributed Systems Platforms (Middleware), pages 329-350, Heidelberg, Germany, Nov. 2001.

[8] S. Saroiu, P. Gummadi, and S. Gribble. A measurement study of peer-to-peer file sharing systems. In Proceedings of Multimedia Computing and Networking (MMCN), San Jones, CA, Jan. 2002.

[9] S. Sen and J. Wong. Analyzing peer-to-peer traffic across large networks. In Proceedings of Second Annual ACM Internet Measurement Workshop, November 2002.

[10] I. Stoica, R. Morris, D. Karger, F. Kaashoek, and H. Balakrishnan. Chord: A scalable peer-to-peer lookup service for Internet applications. In Proceedings of the ACM SIGCOMM 2001, pages 149-160, San Diego, USA, August 2001.

[11] B. Yang and H. Garcia-Molina. Designing a super-peer network. In Proceedings of the 19th International Conference on Data Engineering (ICDE), pages 49-60, Bangalore, India, March 2003.

[12] E. Zegura, K. Calvert, and S. Bhattacharjee. How to model an internetwork. In Proceedings of the IEEE Conference on Computer Communication, pages 594-602, San Francisco, CA, Mar. 1996.

[13] B. Zhao, Y. Duan, L. Huang, A. Joseph, and J. Kubiatowicz. Brocade:landmark routing on overlay networks. In Proceedings of the 1st International Workshop on Peer-to-Peer Systems (IPTPS), Cambridge, MA, March 2002. 